\title{
Mourir de rire, mourir pour rire dans le Quart Livre
}

\section{Christiane Deloince-Louette}

\section{OpenEdition}

Journals

Édition électronique

URL : http://journals.openedition.org/recherchestravaux/267

ISSN : 1969-6434

\section{Éditeur}

UGA Éditions/Université Grenoble Alpes

Édition imprimée

Date de publication : 30 mai 2005

Pagination : 103-112

ISBN : 0151-1874

ISSN : 0151-1874

\section{Référence électronique}

Christiane Deloince-Louette, "Mourir de rire, mourir pour rire dans le Quart Livre », Recherches \&

Travaux [En ligne], 67 | 2005, mis en ligne le 30 septembre 2008, consulté le 07 septembre 2020. URL : http://journals.openedition.org/recherchestravaux/267 


\section{Mourir de rire, mourir pour rire dans le Quart Livre}

Entre l'escale en l'île de Procuration, où demeurent les Chicanous qui gagnent leur vie à être battus, et la terrible tempête que Pantagruel et ses compagnons essuient au large de l'île des Macraéons, le chapitre XVII du Quart Livre mentionne, sans s'y attarder, les îles de Tohu et Bohu. Là, dit le narrateur, "nous ne trouvasmes que frire», le géant Bringuenarilles ayant dévoré toutes les poêles et chaudrons du pays. Ce repas, insolite pour un avaleur de moulins à vent, a provoqué une indigestion qui met le pauvre géant en mauvaise posture. Il mourra "estranglé, mangeant un coin de beurre frais, à la gueule d'un four chauld, par l'ordonnance des Medecins ${ }^{\mathrm{I}}$. Nous ne l'apprendrons qu'à la fin du chapitre, constitué pour l'essentiel d'un catalogue de «morts étranges» empruntées pour la plupart à la compilation de Ravisius Textor $^{2}$. La première version du Quart Livre, parue en I548, énumérait huit cas, dont l'exemple célèbre du poète tragique Eschyle assommé par la carapace d'une tortue qu'un aigle avait lâchée du haut du ciel. À cette liste, Rabelais ajoute deux éléments dans la version définitive de I552: l'exemple de Philémon (Philomenes), poète comique, et celui de Zeuxis, peintre illustre de la belle Hélène. Tous deux morts (littéralement) de rire... devant un spectacle incongru.

Bien des critiques ont tenté d'expliquer ces morts par excès de rire. Mikhail Bakhtine, d'abord, qui y voit une confirmation de l'ambivalence de la mort, dont la potentialité joyeuse est issue de l'idée implicite de rénovation qu'elle contient ${ }^{3}$. Colette Quesnel, tirant parti du chapitre IX de Gargantua

I. J'utilise l'édition de G. Defaux, Paris, Librairie générale française (Bibliothèque classique), 1994 .

2. Les Officina paraissent pour la première fois en 1503 . Le chapitre doit aussi beaucoup à Valère Maxime, Dictorum factorumque memorabilium exempla, IX, I2.

3. M. Bakhtine, L'Euvre de François Rabelais et la culture populaire au Moyen Âge et sous la Renaissance, Paris, Gallimard, 1970 (trad. fr.), p. 406. 
qui cite Philémon parmi les hommes morts de joie, identifie la mort de rire à la mort de joie. Dans la mesure où «le rire, propre de l'homme, est une bénédiction de Dieu", mourir de rire / de joie est l'apanage des héros élevés à la perfection céleste 4 . Discutant cette lecture quelques années plus tard, Daniel Ménager insiste au contraire sur la force destructrice du rire et donc sur l'absurdité des causes qui font mourir's. Plus récemment, Jean-Paul Pagliano justifiait les «morts étranges» par le souci de dérider le lecteur, soulignant que la mort chez Rabelais est vidée de sa dimension horrible ou repoussante ${ }^{6}$.

Or, il faut le souligner, ces deux courts récits de morts de rire sont des additions de 1552 , qu'on peut, pour cette raison, rapprocher d'une autre addition significative: l'épisode des noces de Basché, rapporté par Panurge lors de l'escale en l'île des Chicanous, épisode qui renferme, comme en abyme, la «tragicque farce» jouée par François Villon au détriment d'Étienne Tappecoue, «secretain des Cordeliers», qui a refusé de prêter aux comédiens "chappe et etole». La farce s'achève par la mort atroce de Tappecoue, dépecé par la course effrénée de sa jument. Villon, lui, jubile et félicite ses «diables» de la qualité de leur jeu. C'est Panurge qui rapporte l'épisode, Panurge lui-même metteur en scène d'une autre "tragicque farce", la mort grotesque du marchand Dindenault, noyé avec ses moutons et ses bergers (chapitres V à VIII). Des morts pour rire donc, propres à réjouir leur public, Panurge certes mais aussi Frère Jan, spectateur attentif du "passe-temps» (dans l'épisode de Dindenault) ou auditeur intéressé (dans le récit des noces de Basché).

Mourir pour rire, mourir de rire: trois additions congruentes du Quart Livre de 1552 invitent donc à repenser, dans cette œuvre, les liens entre la mort et le rire. D'une part, dans le contexte du jeu carnavalesque; d'autre part, dans l'organisation interne de l'œuvre et, plus exactement, dans la perspective

4. C. Quesnel, Mourir de rire d'après et avec Rabelais, Cahiers d'études médiévales, $\mathrm{n}^{\circ}$ Io, Montréal, Bellarmin, Montréal/Paris, Vrin, I99I, p. 9I-93. Voir Gargantua, éd. G. Defaux, Paris, Librairie générale française I994, chap. IX, p. I57-I59.

5. La Renaissance et le rire, Paris, Presses universitaires de France, 1995, p. 27. Une telle lecture se rapproche de la conception des novellistes italiens du XVe siècle, pour qui le rire, y compris dans son association avec la mort, «devient [...] la mesure humaine, le mètre narratif de la variété, de la labilité, de la réversibilité: il est comme le révélateur ou la prise de conscience renouvelée que le monde n'est pas un et stable». (J. Lacroix, "Esquisse d'une signification du rire chez les nouvellistes italiens des XIII", XIVe et XVe siècles", Le Rire au Moyen Âge, textes recueillis par Th. Bouché et H. Charpentier, Presses universitaires de Bordeaux, I990, p. 220). J. Lacroix rappelle également que, dans le Morgante de Pulci, le demi-géant Margutte éclate littéralement de rire à la vue d'un singe qui avait chaussé ses houseaux (chant XIX, str. I48-I49).

6. J.-P. Pagliano, «Remarques sur les morts étranges dans l'œuvre de Rabelais et la fonction du rire", Ec-Rire, Actes du colloque international sur le rire, le comique et l'humour, Pécs, université de Pécs, 200I. 
ouverte par le prologue et sa leçon: «souhaitez donc médiocrité». Au cœur de cette enquête, l'articulation de deux concepts apparemment inconciliables: l'excès carnavalesque et la «médiocrité» évangélique.

\section{Morts de rire!}

Parmi les morts étranges du chapitre XVII, le récit de la mort de Philomenes (en réalité l'auteur comique Philémon) est le plus étoffé.

Plus de Philomenes, auquel son varlet, pour l'entrée de dipner, ayant apresté des figues nouvelles, pendent le temps qu'il alla au vin, un asne couillart esguaré estoit entré on logis, et les figues apposées mangeoit religieusement. Philomenes survenent, et curieusement contemplant la grace de l'asne Sycophage, dist au varlet qui estoit de retour: «Raison veult, puys qu'à ce devot asne as les figues abandonné, que pour boire tu luy produise de ce bon vin que as apporté.» Ces parolles dictes, entra en si excessive guayeté d'esprit, et s'esclata de rire tant enormement, continuement, que l'exercice de la Ratelle luy tollut toute respiration et subitement mourut. ${ }^{7}$

Ce récit, très connu dans l'Antiquité, où il est parfois attribué au stoïcien Chrysippe, Rabelais a pu le trouver chez Valère Maxime ou dans la compilation de Ravisius Textor, peut-être aussi chez Lucien dans un opuscule aujourd'hui considéré comme apocryphe, mais dont on ne lui disputait pas, au XVI ${ }^{e}$ siècle, la paternité: les «Longue-vie» ou Macrobioi ${ }^{8}$.

Le rire de Philomène est provoqué à la fois par un spectacle comique voir l'âne manger les figues - et par la plaisanterie qui en jaillit - qu'on lui donne aussi à boire! Rabelais cependant réécrit ses sources: Philémon ne rit plus seulement de voir un âne manger son dîner, mais de la congruence inattendue de certains éléments. L'âne rabelaisien est d'abord couillart; cette

7. Op. cit., p. 297. Dans Gargantua, Philémon est mentionné non seulement au chap. IX mais au chap. XIX (éd. citée, p. 217).

8. Voici le récit rapporté par Lucien, Macrobioi, 25: «Philémon le comique est comparable à Cratinos : ayant vécu quatre-vingt-dix-sept ans, il se reposait étendu sur un lit quand il vit un âne dévorer les figues préparées pour lui; il éclata de rire et, appelant son esclave, il lui dit, en riant beaucoup et de toutes ses forces, de donner aussi du vin pur à boire à l'âne; il s'étouffa à force de rire et mourut. " Lucien, Euvres, t. II, Paris, Les Belles Lettres, trad. J. Bompaire. Valère Maxime insiste encore davantage sur le caractère excessif du rire: Philemonem autem vis risus immoderati abstulit: paratas ei ficus, atque in conspectu positas asello consumente, puerum, ut illum abigeret, inclamavit; qui quum jam comestis omnibus supervenisset; "Quoniam, inquit, tam tardus fuisti, da nunc merum asello"; ac protinus urbanitatem dicti crebro anhelitu cachinnorum prosequuntus, senile guttur salebris spiritus praegravavit. (IX, XII, 6), éd. et trad. Nisard, Paris, Dubuchet et Cie, I84I.

Rappelons cependant que c'est justement un «Macrobe» que les héros rencontrent dans l'île des Macraéons après la tempête en mer, épisode qui commence au chap. XVIII. L'édition de I548 s'arrête abruptement au moment où les compagnons arrivent dans l'île des Macraéons. La rencontre du «Macrobe» n'existe que dans la version de 1552. 
précision impose un rapprochement significatif entre la mort de Philémon et l'épisode des Papefigues (chapitre XLV) où la figue est clairement un symbole sexuel 9 . La version de Rabelais accentue le comique du bas corporel: manger des figues devient métaphore sexuelle. Or l'âne couillart est aussi dévot, il mange les figues religieusement. On retrouve la veine satirique médiévale à l'encontre des moines, paillards et gourmands, mais surtout, comme en filigrane, la fête carnavalesque de l'âne: l'âne, couillart et dévot, célèbre ici une messe parodique, sous les deux espèces des figues et du vin. Dans ce contexte, le rire aussi est carnavalesque: il est participation à un renversement du monde, à une parodie jouissive des gestes et rites liturgiques, autant qu'à une satire de la pseudo-dévotion des religieux, plus sensibles à l'attrait de la figue qu'à celui de l'hostie. Or, ce sont les paroles de Philomenes lui-même, qui nous incitent à cette interprétation. Autrement dit, le poète comique rit aussi de son esprit, de sa capacité à tirer un bon mot d'un spectacle. L'excès de rire semble être causé par une forme excessive d'amour de soi, ce vice qu'Érasme nomme "philautie ${ }^{\mathrm{IO}}$ ».

À Zeuxis, Rabelais ne consacre qu'une phrase:

Plus de Zeusis le painctre, lequel subitement mourut à force de rire, considerant le minoys et portraict d'une vieille par luy representée en paincture.

Il a pu trouver l'anecdote chez Érasme, dans l'adage intitulé Risus sardonicus (III, V, I I II. Si le rire est également qualifié d'excessif, le comique est plus difficile à percevoir: Zeuxis était célèbre pour son Hélène, parangon de toute beauté, ou pour ses grappes de raisin que les oiseaux venaient picorer, les croyant vraies. La clé de l'énigme se trouve peut-être chez Aristote qui associe le rire et la laideur. Le peintre de la belle Hélène aurait ri de sa capacité à représenter son contraire, la vieille femme laide. Si la notion de laideur n'est pas explicite, elle est sous-entendue dans l'emploi du terme «minoys", très

9. Les Papefigues sont ainsi nommés parce qu'ils ont «fait la figue» au pape. Cruellement battus par les Papimanes, ils durent, pour avoir la vie sauve, satisfaire à «condition semblable à celle dont l'empereur Federic Barberousse jadis usa envers les Milanois»: saisir à pleines dents une figue stratégiquement placée "les membres honteux" d'une mule dont le nom, Thacor désigne en hébreu, d'après la Briefve Declaration, "un fic au fondement" - fic signifiant à la fois verrue et figue (Le Quart Livre, éd. citée, p. 468-469 et p. 640 pour la Briefve Declaration). Le sens sexuel de figue est déjà attesté, en grec, chez Aristophane, La Paix, v. I349.

Io. Ce que soulignait déjà D. Ménager, op. cit., p. 29.

II. Le récit est très succinct: Mortuus est et Zeuxis pictor ridendo, dum sine fine ridet anum a se pictam (Adagia, III, V, I; Opera omnia, t. V, North Holland Publishing Company, Amsterdam-Oxford, 198I). Érasme mentionne aussi l'âne mangeur de figues mais attribue l'anecdote à Chrysippe, suivant en cela Diogène Laërce. 
péjoratif au XVI ${ }^{\mathrm{e}}$ siècle ${ }^{\mathrm{I}}$. Le couple vieillesse-laideur est du reste bien attesté dans l'Antiquité, chez Horace (Odes, I, 25; III, I5; IV, I3 ou, pire encore, Épodes, VIII) et Juvénal (satire X). Si le rire provient ici encore d'un spectacle comique, il participe aussi, comme dans le cas de Philémon, du rire satirique. Il le dépasse pourtant, par son excès même: Zeuxis aurait-il découvert subitement une forme d'identité entre la laideur et la beauté? $\mathrm{Ou}$, dans une perspective carnavalesque, peindre la laide vieillesse après la belle jeunesse ne serait-il pas le signe d'un vieillissement du peintre, lisant dans son tableau, comme dans un miroir, la preuve qu'il a fini son temps? La mort par excès de rire reste une mort carnavalesque, la mort joyeuse des représentants d'un ancien monde (chez Lucien, rappelons-le, Philémon est un "Macrobe»: il n'est donc plus tout jeune).

Il ne faut donc pas s'empresser de considérer la mort de rire comme le châtiment d'une forme d'amour de soi. Si elle peut être liée à la complaisance que l'artiste manifesterait à l'égard de son discours ou de son œuvre, elle reste avant tout l'expression de la joyeuse disparition d'hommes qui ont fait leur temps: qui ne souhaiterait mourir dans un éclat de rire?

\section{Rire de la mort: la «tragicque farce»}

Les deux mises à mort de Dindenault et de Tappecoue s'inscrivent d'abord dans un contexte carnavalesque. L'épisode fameux des «moutons de Panurge» occupe les chapitres V à VIII. Panurge, traité de cocu par le marchand sûr de lui, organise une vengeance spectaculaire, offerte à un public choisi : frère Jan et Épistémon ${ }^{13}$. Après avoir supporté patiemment les avanies et injures de Dindenault, il feint de vouloir acheter un mouton. Mais, sitôt payé, le mouton est jeté à la mer. À sa suite se précipitent, dans l'ordre, le reste du troupeau, les bergers, le marchand: "Tous furent pareillement en mer portez et noyez miserablement». Non content de l'heureuse issue, Panurge, l'aviron en main, assomme ceux qui veulent grimper sur le navire et les félicite joyeusement de quitter enfin notre vallée de larmes. Frère Jan, pris à témoin de l'habileté du rusé, ne lui reproche qu'une chose: avoir payé le mouton!

Le récit de la mort d'Étienne Tappecoue est inséré dans l'épisode plus ample des noces de Basché, procédé mis au point par le seigneur de Basché

I2. Le dictionnaire de E. Huguet rappelle le sens du terme dans les Épithètes de M. de la Porte: visage "chagrin, tetrique, rude, mal plaisant", termes qui reprennent au reste l'évocation de la mine chagrine du mauvais médecin dans l'épître à Odet de Chastillon qui ouvre Le Quart Livre (éd. citée, p. 133).

I3. Panurge leur dit «secretement»: «Retirez vous icy un peu à l'escart, et joyeusement passez temps à ce que voirez. Il y aura bien beau jeu, si la chorde ne rompt.» (chap. VI, éd. citée, p. 213) Remarquons l'absence de Pantagruel, héros évangélique dans Le Quart Livre. 
pour se débarrasser de Chicanous encombrants: recourant à la coutume des «noces à mitaines» où les invités échangent coups et bourrades pour fêter les nouveaux mariés, Basché, à l'arrivée des huissiers, feint de célébrer des noces. Les Chicanous, dûment festoyés, puis battus, s'en retournent dolents mais joyeux. Pour fêter le succès de sa ruse, Basché rapporte à ses domestiques l'histoire de Tappecoue. Étienne Tappecoue, sacristain imbu de sa personne, respectant à la lettre les statuts provinciaux, a refusé à Villon le prêt d'ornements sacerdotaux pour la mise en scène d'un mystère de la Passion. La vengeance sera sanglante: les «diables», masques de carnaval,

[...] tous capparassonnez de peaulx de loups, de veaulx et de beliers, passementées de testes de mouton, de cornes de bøufz et de grands havets de cuisine

font un vacarme si épouvantable sur le passage du sacristain que sa jument s'emballe: jeté à bas de sa monture mais retenu par son soulier, le pauvre Tappecoue est littéralement mis en pièces, déchiqueté comme le Penthée des Bacchantes d'Euripide ${ }^{\mathrm{I}}$. La "tragicque farce» ainsi achevée, loin de déplorer l'issue lamentable de la ruse, Villon félicite ses diables: «O que vous jourrez bien!». La mise à mort illustre paradoxalement la perfection du jeu des acteurs.

De Dindenault à Tappecoue, la continuité semble évidente: les traquenards mortels tendus au marchand comme au sacristain sont mises à mort "pour rire» des épouvantails du carnaval. Elle est condamnation farcesque d'hommes prétentieux et vaniteux, persuadés de détenir l'autorité. Point commun supplémentaire: dans les deux cas, la présence de Panurge accompagne le succès d'une ruse cruelle mais joyeuse. C'est du moins l'opinion de Panurge lui-même.

Or l'attitude de Pantagruel oblige à nuancer cette première lecture. Le récit de Panurge provoque chez lui une autre réaction que la joie:

Cette narration [...] sembleroit joyeuse, ne feust que davant nos oilz fault la craincte de Dieu continuellement avoir. ${ }^{\text {Is }}$

Ce jugement évangélique fait écho à la mise en garde de frère Jan, disant à Panurge, à l'extrême fin du chapitre VIII:

Tu te damnes comme un vieil diable. Il est escript: Mihi vindictam, et caetera. Matiere de breviaire. ${ }^{16}$

I4. M. Bakhtine rappelle à ce sujet l'expression carnavalesque de "ragoût de Penthée», op. cit., p. 267.

I5. Chap. XVI, p. 287.

I6. Ibid., p. 23I. Frère Jan cite les premiers mots du chap. I2, verset I9, de l'épître aux Romains: «À moi la vengeance: c’est moi qui rétribuerai, dit le Seigneur." 
Pourquoi ces réticences? C'est que Panurge, comme Villon, détourne le contexte carnavalesque au service d'une vengeance personnelle. Mettre à mort les vieux rois, la chose est certes louable, mais dans un cadre de fête collective ou, ce qui revient au même, dans les limites d'une conception évangélique du monde.

C'est dans cette perspective que l'on pourrait reconsidérer les morts de rire, dont les récits apparaissent comme l'inverse des farces de Dindenault et de Tappecoue. Aux deux épisodes qui mettent en scène, "pour rire", des morts cruelles, répondent deux anecdotes où un spectacle comique provoque la mort par excès de rire. Dans le premier cas, ce sont les personnages qui meurent, pour le plus grand plaisir du metteur en scène et des spectateurs / lecteurs ; dans le second cas, ce sont les metteurs en scène, ou plus exactement les auteurs du spectacle (paroles de Philémon, auteur comique et tableau de Zeuxis, peintre) qui meurent par excès de rire. Les deux ensembles se répondent et s'opposent, comme se corrigeant mutuellement. L'hypothèse serait donc la suivante: Rabelais, dans sa version définitive, aurait établi cette symétrie de façon à compenser l'excès de cruauté des farces tragiques. Le but est-il moral ? Pas exactement. Il trouve sa justification dans le prologue de l'édition de 1552 .

\section{Souhaitez donc médiocrité}

Au centre du prologue, l'apologue ésopique du bûcheron Couillatris. Ayant malencontreusement perdu sa cognée, il harcèle de ses cris le maître de l'Olympe. Pour s'en débarrasser, Jupiter charge Mercure de proposer au pauvre bûcheron trois cognées: l'une est d'or, l'autre d'argent, la troisième n'est que la sienne. Avec joie, Couillatris retrouve la sienne et, pour prix de sa «mediocrité», Mercure lui offre les deux autres. Et ses voisins, envieux, de perdre à leur tour leurs cognées et de crier vers Jupiter. Mais tous ne sont pas de «médiocres» Couillatris. À ces hommes cupides qui se précipitent sur la cognée d'or, Mercure coupe la tête sans état d'âme. À l'excès de prétention répond l'excès de cruauté: nous retrouvons un schéma analogue à celui des épisodes de Dindenault et de Tappecoue.

Dans l'économie du prologue de 1552 , la "médiocrité» est évoquée en tant que qualité évangélique:

J'ay cestuy espoir en Dieu qu'il oyra nos prieres, veue la ferme foy en laquelle nous les faisons; et accomplira cestuy nostre soubhayt, attendu qu'il est mediocre. (p. 159)

Comme le petit Zachée de l'Évangile, qui ne voulait que voir le Christ, comme Élisée, comme Couillatris ensuite, le lecteur est joyeusement invité à borner ses désirs: 
Soubhaitez donc mediocrité: elle vous adviendra, et encores mieulx, deument ce pendent labourans et travaillans (p. 175)

La médiocrité antique, ou modération, devient l'équivalent de l'humilité évangélique, les "sages anciens" sont cités à côté de la Bible ${ }^{17}$.

Or, pour Érasme, grand admirateur de Lucien et père spirituel de Rabelais, la modération relève de la connaissance de soi. L'adage 595, Nosce te ipsum, souligne en effet:

In quo modestia mediocritatisque commendatio est, ne nobis vel maiora vel etiam indigna sequamur. Nam hinc omnis vitae pestis oritur, quod sibi quisque blanditur et quantum aliis praeter aequum detrahit, tantum sibi philautiae vitio praeter meritum tribuit. ${ }^{18}$

Le refus de la modération ouvre la porte à la «philautie». Nous retrouvons la leçon de Pantagruel devant le double récit de Panurge:

Cette narration $[\ldots]$ sembleroit joyeuse, ne feust que davant nos oilz fault la craincte de Dieu continuellement avoir.

En utilisant, à des fins de vengeance personnelle, le jeu et le rire carnavalesques, Panurge et Villon ont dévoilé leur philautie, qu’on peut alors définir comme le souci excessif de soi et de son intérêt, au détriment de la crainte de Dieu. À la joie mauvaise de Panurge ou de Villon, se félicitant du succès de leur farce, Pantagruel oppose ici, implicitement, la vraie joie évangélique et pantagruélique, celle de l'apôtre Paul qui, dans la première épître aux Thessaloniciens, invite ses lecteurs à «vivre toujours joyeux» $(5, \mathrm{I} 6)$.

La mort de rire relève tout aussi bien à la fois de la verve carnavalesque et, bien que moins nettement, d'une mise en garde contre le vice de philautie. Il est sans doute vain de chercher à l'interpréter pour elle-même. En revanche, si l'on considère la disposition des épisodes étudiés, les deux anecdotes du

I7. À côté d'Horace et de sa mediocritas aurea (Odes, II, IO, 5), c'est encore Lucien que l'on retrouve. Les premières lignes de son Zeus confondu mettent en scène Cyniscos et Zeus qui souligne l'importance des souhaits modérés:

«Cyniscos: Mais moi, Zeus, je ne t'ennuierai pas avec ce genre de demandes, richesse, or ou royauté, qui sont l'objet des prières ardentes de la plupart des gens et que tu as bien du mal à procurer. En tout cas je vois que la plupart du temps tu ne prêtes pas l'oreille à leurs prières. Il est une seule chose - et facile à réaliser - que je voulais obtenir de toi.»

Zeus: De quoi s'agit-il, Cyniscos? Car tu ne seras pas déçu, surtout si ta requête est modérée, comme tu le disais. (Lucien de Samosate, Euvres, t. II, Paris, Les Belles Lettres, I999).

I8. Éd. citée, t. I: «Dans cet adage, la mesure est recommandation de modération : il faut éviter d'avoir des désirs trop élevés ou même indignes. Car c'est la cause de la ruine tout entière de l'existence: chacun se flatte lui-même et tout ce qu'il enlève aux autres contre l'équité, il se l'attribue, par vice de philautie, contre son mérite.» (Notre traduction) L'adage 596, Ne quid nimis, est nourri de la même réflexion. 
chapitre XVII semblent jouer le rôle d'un contrepoint aux mises à mort comiques (toutes les deux antérieures dans le récit). Rabelais ne condamne explicitement ni Panurge ni Villon, mais il propose au lecteur une mise en scène inversée qui en sanctionne implicitement les excès, par un rire luimême excessif.

Loin d'abandonner la verve carnavalesque au profit d'une leçon évangélique, Rabelais aurait donc recours à cette même technique du contrepoint qu'il a déjà pratiquée dans Gargantua ${ }^{19}$. Compenser l'excès par l'excès inverse. Ce qui lui permet de laisser la porte ouverte à une lecture évangélique sans pour autant l'énoncer de manière dogmatique et rigide. Entre deux excès, choisissez donc «mediocrité».

\section{La figue et la cognée}

Alors comment faut-il rire? Comme les dieux, et comme les mouches, nous dit Rabelais dans un raccourci burlesque. Couillatris réclame sa cognée? Mais, demande Priape, «le roide dieu des jardins» : compte tenu de l'équivocité du mot cognée qui signifie tout à la fois «un certain instrument, par le service duquel est fendu et couppé boys» et «la femelle bien à poinct et souvent gimbretiletolletée»,

[...] ores seroit à sçavoir quelle espece de coingnée demande ce criart Couillatris.

Â ces motz, tous les venerables Dieulx et Deesses s'eclaterent de rire comme un microcosme de mouches. (Prologue, p. 167)

Par un bon mot, Priape déride Jupiter, fort préoccupé par les affaires en cours et peu soucieux de Couillatris. Le rire joyeux est donc un rire facétieux, analogue semble-t-il à celui de Lucien tel que le définit Érasme: "[...] nec illiberalis, nec a mouso $[\ldots]^{20}$ ». Un rire sans bassesse et non étranger aux Muses. Un rire qui, surtout, est l'expression non d'une satisfaction égoïste, mais du désir de partager une joie.

Un tel rire sera celui du médecin dont «la face joyeuse, seraine, gratieuse, ouverte, plaisante, resjouist le malade» et lui permet d'augurer favorablement

19. Voir F. Rigolot, Les Langages de Rabelais, Genève, Droz (Titre courant), 1996, qui souligne «le jeu des thèses opposées" à propos, par exemple, des deux pédagogies à l'œuvre dans Gargantua: la pédagogie scolastique et la pédagogie humaniste sont toutes deux placées sous le signe de l'excès, repérable stylistiquement. Rabelais les renvoie dos à dos, stigmatisant les tendances excessives de l'une comme de l'autre. Rappelons, dans le même esprit, la règle aristotélicienne du juge Bridoye: opposita, juxta se posita, magis elucescunt, "En plaçant côte à côte les termes opposés, on les voit plus clairement» (Le Tiers Livre, éd. J. Céard, Paris, LGF, 1995, chap. XXXIX, p. 37I).

20. Lettre à Guillaume Warham, Allen, II, 293, cité par Ch. Lauvergnat-Gagnière, Lucien de Samosate et le lucianisme en France au XVI e siècle, Genève, Droz, 1988, p. 233. 
de sa guérison ${ }^{21}$. Ce sera aussi, sans doute le rire de l'écrivain, soucieux de partager avec ses lecteurs les joies du «Pantagruélisme (vous entendez que c'est certaine gayeté d'esprit conficte en mespris des choses fortuites) ${ }^{22}{ }$ Cette même gaieté d'esprit qui doit saisir le lecteur à la lecture d'un bon mot. Quand Priape équivoque sur le mot «cognée», Rabelais joue du double sens de «figue». L'équivoque obscène ou grivoise qui provoque l'éclat de rire partagé unit dans une même joie la gaieté carnavalesque et la convivialité évangélique. Ce n'est pas un des moindres paradoxes de Rabelais.

2I. Épître au cardinal Odet de Chastillon, éd. citée, p. 135.

22. Prologue, op. cit., p. I49. 